usual algebras there should be greater care. Thus, on page 38 , line $4, \rho^{2}$ should be replaced by $\rho$. On page 41 , the sum of $a=\left(\alpha_{1}, \alpha_{2}\right)$ and $b=\left(\beta_{1}, \beta_{2}\right)$ is of course not $\left(\alpha_{1}+\alpha_{1}, \beta_{1}+\beta_{2}\right)$, but $\left(\alpha_{1}+\alpha_{2}, \beta_{1}+\beta_{2}\right)$.

Excessive brevity, especially toward the end, is hard on the reader. Equality of $\mathfrak{Y}$ vectors in an $\mathfrak{Y} \mathfrak{B} \mathfrak{E}$ web (Vektorgleichheit) and in an $\mathfrak{Y} \mathfrak{B} \mathfrak{P}$ web (Masszahlengleichheit) do not mean the same thing, yet they are both called equality; this is especially confasing when, as on page 117, all four pencils are named. And, finally, it is to be hoped that the author will supply the next edition with an index and with an appendix containing all the axioms, to which reference must frequently be made.

Both books are excellently printed. Springer draws attention to his clear, neat page by a footnote on page 130 in contrasting type.

A good geometrical library will certainly wish to possess both works, the more so since they so surprisingly avoid duplication of subject matter. If a reader studies first the Hessenberg work, with its appeal to the pictorial sense, and then the sterner, very stimulating book of Reidemeister, he can gain greatly in his knowledge of the logic of geometry.

\title{
E. S. Allen
}

\section{FOU R FRENCH BOOKS ON HYDRODYNAMICS}

(1). Leçons sur la Hydrodynamique. By H. Villat. Paris, Gauthier-Villars, 1929. iii $+296 \mathrm{pp}$.

(2). Lę̧ons sur la Théorie des Tourbillons. By H. Villat. Paris, Gauthier-Villars, 1930. ii $+300 \mathrm{pp}$.

(3). Mécanique des Fluides. By H.Villat. Paris, Gauthier-Villars, 1930.vii +175pp.

(4). Lę̧ons sur la Résistance des Fluides non Visqueux. By P. Painlevé. Première partie rédigée par A. Metral. Paris, Gauthier-Villars, 1930.iv+183 pp.

Within the last decade or two the interest in research in hydrodynamics has spread widely throughout all European countries, almost without exception, and very important progress is being achieved in various branches of theoretical and applied hydrodynamics. It is the more striking and disappointing that America has practically no share in this recent progress of hydrodynamics, at least as far as theory is concerned. The amount of papers on theoretical hydrodynamics published in this country is practically negligible. This is deplorable not only because of the high intrinsic interest and practical value of hydrodynamics, but also because in hydrodynamics we have an excellent field of application and means of sharpening of various mathematical tools. Suffice it to say that the recent progress of hydrodynamics mentioned above is intimately related to the modern developments of the theory of functions of a complex variable, conformal mapping, potential theory, integral and integrodifferential equations and the like, being in many cases not only the after-effect but even the cause of the development. The last but not the least important aspect of hydrodynamics is the pedagogical one: there is perhaps no better way of lliustrating abstract mathematical theories and of developing in students a critical ability in applying and interpreting mathematical results. It is to be hoped that the recent books published in Germany (by Oseen and Lichtenstein) 
and in France (books under review) will awaken interest in hydrodynamics and will place hydrodynamical research in this country on a fitting level.

The modern progress of hydrodynamics is well reflected in the books under review, at least in those by $\mathrm{H}$. Villat. They are devoted to the hydrodynamics of an incompressible (perfect or viscous) fluid, of which the irrotational motion is discussed in (1), (2) being concerned primarily with the theory of vortices.

In Chapters 1-4 of (1) we have an excellent exposition of some indispensable results of the theory of functions of a complex variable and conformal mapping. We note particularly the elegant introduction of elliptic functions in connection with the solution of Dirichlet's problem for a circular ring between two concentric circles, and explicit formulas for the conformal mapping of a simplyconnected domain on the unit circle (due to Cisotti); these formulas are applied to a derivation of the classical Schwarz-Christoffel formulas for the mapping of polygons. After recalling the fundamental equations of motion of perfect fluids (Chapter 5), we pass on to a detailed discussion of the theory of liquid jets (originated by Helmholtz and Kirchhoff), Chapters 6-8. Investigations of several writers, those of the author himself inclusive, are discussed here in a very clear fashion. Various difficulties in the application of abstract results are pointed out and some remarkable cases of the multiplicity of physically admissible solutions are treated. The rest of the book is devoted to problems of motion of a viscous (incompressible) fluid, mainly to that of the motion of a rigid body in such a fluid. The author succeeds completely in the difficult task of giving a rapid but clear and accessible exposition of the complicated theory developed by Oseen and his pupils, particularly by Zeilon. The most important problem of this theory is the discussion of liquids with small viscosity and the investigation of the limiting solutions obtained when the coefficient of viscosity tends to zero. This is done in Chapters 14-16, on the basis of Oseen's integral equations (Chapter 13), after the equations of a viscous fluid have been derived (Chapter 9 ) and certain important properties of fundamental solutions of the heat equation (Chapter 11) and of Newtonian potentials (tangential derivatives of potentials of a simple spread, Chapter 12) have been established. An important result of the theory of Oseen-that the motion of a perfect fluid is not the limiting case of the motion of a viscous fluid whose coefficient of viscosity tends to zero-had already been observed in a very special case by Boussinesq. The analysis of this case (Chapter 10) helps the reader considerably in the understanding of the much more general theory of Oseen. The concluding Chapters 17-20 are given to the treatment of special cases, in two and three dimensions, on the basis of papers by Zeilon. An elegant application of properties of Legendre's functions is pointed out in Chapter 19.

Book (2), as follows from its title, treats of the theory of vortices and turbulent motion. In Chapters 1-2 general equations are derived and formulas expressing the velocities in terms of the vortices are given. Chapters 3-6 deal with problems arising in the theory of plane motion of rectilinear vortex lines: canonical equations, stability questions, vortices of Kármán-Bénard and their various extensions, computation of the resistance of the liquid due to turbulent motion. Interesting remarks concerning applications of conformal mapping to the problems of vortex motion in two dimensions are found in Chapter 7. Chapter 8 treats of some special problems of vortices of finite dimensions 
(elliptic vortex of Kirchhoff and spherical vortex of Hill), while vortex rings (of infinitely small section) are discussed in Chapter 9. Chapter 10 recalls some fundamental facts of the theory of propagation of discontinuities, due mainly to Hadamard. Chapters 11-13 are devoted to a brief exposition of the fundamental existence theorem for the equations of hydrodynamics. It is rather unfortunate that the method used here is ascribed to Lichtenstein alone, whose name only is mentioned in the book; no reference is made to the important work of Günther, which deserves as much credit as that of Lichtenstein. The concluding Chapter 14 deals with some special cases of vortices in a viscous fluid. It is seen from this rapid survey that several important problems of hydrodynamics, both classical and modern, are not touched upon in the two excellent monographs of $\mathrm{H}$. Villat, for example, figures of equilibrium of celestial bodies, wave-motion, hydrodynamics of compressible fluids and of those with radiation of energy, including the dynamics of atmosphere, statistical methods. While this appears to be quite natural and wise, on the whole, it is the reviewer's opinion that the important investigations of Friedmann concerning the kinematics of vortices of fluids that do not obey Helmholtz's laws should be treated in a more detailed way than is actually done in Chapter 1. Problems of turbulent motion also might have been treated in more detail, and a brief exposition of statistical methods would not be out of place in a treatise on modern hydrodynamics: it is by these methods that, in all likelihood, a true theory of resistance of fluids will be found, and mysteries of the dynamics of atmosphere will be explained.

Book (3) is of a more elementary and less inclusive character than the preceding two, with which it overlaps in greater part. As interesting additions we should mention, however, an excellent account of the Kutta-Joukowsky Theorem with its applications and discussion of cases of exception, and an exposition of Prandtl's theory of carrying surfaces. All three books by H. Villat under review are marked by their clear and suggestive style of exposition. The misprints, although rather frequent, do not spoil appreciably the pleasure of reading these books. We are looking forward to the promised continuation of these monographs, Sur la Répresentation Conforme et quelques unes de ses Récentes Applications, which is now in press.

Book (4) by P. Painlevé is on a level quite different from those by $\mathrm{H}$. Villat, both as to choice of material and exposition. Out of its 183 pages only 88 treat of hydrodynamical subjects at all, the remaining space being given to an outline and mathematical preparations. The most interesting part of the book is the "préambule" (on theoretical hydrodynamics and experimental laws of air resistance), incomplete as it is. The exposition of other chapters is confined to generalities that might be found in any standard treatise on dynamics of fluids, without illustration by concrete examples. The misprints and lapses, corrected in the Errata, as well as uncorrected ones, are abundant. The final judgment about Painlevé's Lę̧ons should be reserved until the publication of their second part, the content of which is unknown at present. It is probably there that the author expects to succeed in his efforts to "pousser aussi loin que possible la solution théorique de ce grand problème" (rigorous solution of the problem of a motion of a rigid body in a perfect liquid).

J. D. TAMARKIN 